\title{
Bayesian Inference for Gravitational Waves from Binary Neutron Star Mergers in Third Generation Observatories
}

\author{
Rory Smith $\odot,{ }^{1,2, *}$ Ssohrab Borhanian $\odot,{ }^{3, \dagger}$ Bangalore Sathyaprakash $\odot,{ }^{3,4,5, *}$ Francisco Hernandez Vivanco, ${ }^{1,2}$ \\ Scott E. Field, ${ }^{6}$ Paul Lasky $\odot,{ }^{1,2}$ Ilya Mandel, ${ }^{1,2}$ Soichiro Morisaki, ${ }^{7}$ David Ottaway, ${ }^{8}$ Bram J. J. Slagmolen, ${ }^{9}$ \\ Eric Thrane, ${ }^{1,2}$ Daniel Töyrä, ${ }^{9}$ and Salvatore Vitale $\odot^{10,11}$ \\ ${ }^{1}$ School of Physics and Astronomy, Monash University, Victoria 3800, Australia \\ ${ }^{2}$ OzGrav: The ARC Centre of Excellence for Gravitational Wave Discovery, Clayton, Victoria 3800, Australia \\ ${ }^{3}$ Institute for Gravitation and the Cosmos, Department of Physics, Pennsylvania State University, \\ University Park, Pennsylvania 16802, USA \\ ${ }^{4}$ Department of Astronomy and Astrophysics, Pennsylvania State University, University Park, Pennsylvania 16802, USA \\ ${ }^{5}$ School of Physics and Astronomy, Cardiff University, Cardiff CF24 3AA, United Kingdom \\ ${ }^{6}$ Department of Mathematics and Center for Scientific Computing and Visualization Research, \\ University of Massachusetts, Dartmouth, Massachusetts 02747, USA \\ ${ }^{7}$ Department of Physics, University of Wisconsin-Milwaukee, Milwaukee, Wisconsin 53201, USA \\ ${ }^{8}$ OzGrav, University of Adelaide, Adelaide, South Australia 5005, Australia \\ ${ }^{9}$ OzGrav, ANU Centre for Gravitational Astrophysics, Research Schools of Physics, and Astronomy and Astrophysics, \\ The Australian National University, Australian Capital Territory 2601, Australia \\ ${ }^{10}$ LIGO Laboratory, Massachusetts Institute of Technology, Cambridge, Massachusetts 02139, USA \\ ${ }^{11}$ Department of Physics and Kavli Institute for Astrophysics and Space Research, Massachusetts Institute of Technology, \\ 77 Massachusetts Avenue, Cambridge, Massachusetts 02139, USA
}

(Received 29 March 2021; revised 20 June 2021; accepted 9 July 2021; published 20 August 2021)

\begin{abstract}
Third generation (3G) gravitational-wave detectors will observe thousands of coalescing neutron star binaries with unprecedented fidelity. Extracting the highest precision science from these signals is expected to be challenging owing to both high signal-to-noise ratios and long-duration signals. We demonstrate that current Bayesian inference paradigms can be extended to the analysis of binary neutron star signals without breaking the computational bank. We construct reduced-order models for $~ 90$-min-long gravitational-wave signals covering the observing band (5-2048 Hz), speeding up inference by a factor of $\sim 1.3 \times 10^{4}$ compared to the calculation times without reduced-order models. The reduced-order models incorporate key physics including the effects of tidal deformability, amplitude modulation due to Earth's rotation, and spin-induced orbital precession. We show how reduced-order modeling can accelerate inference on data containing multiple overlapping gravitational-wave signals, and determine the speedup as a function of the number of overlapping signals. Thus, we conclude that Bayesian inference is computationally tractable for the long-lived, overlapping, high signal-to-noise-ratio events present in $3 \mathrm{G}$ observatories.
\end{abstract}

DOI: 10.1103/PhysRevLett.127.081102

Introduction.-Third generation (3G) gravitationalwave detectors such as Cosmic Explorer (CE) [1] and the Einstein Telescope (ET) [2] will observe hundreds of thousands to millions of binary neutron star (BNS) mergers a year [3,4]. Many of the observed signals will be extremely loud, with signal-to-noise ratios (SNRs) $\sim O(100-1000)$. These signals will provide exquisite measurements of neutron star masses, tidal deformability, and spins, facilitating breakthroughs in cosmology and fundamental physics [5,6]. Analyzing signals in the $3 \mathrm{G}$ era will require scaling data analysis methods by orders of magnitude beyond their current capabilities: Signals will be in band up to around 40 times longer than in Advanced LIGO and Virgo, the event rate will be thousands of times higher, and multiple signals will be in band at any one time $[1,2]$.
Bayesian inference is the gold standard for measuring the properties of gravitational-wave signals [7-10]. In Bayesian inference, the posterior probability density of source parameters $\Theta$ given experimental data $d$ and a hypothesis for the data $\mathcal{H}$ is

$$
p(\Theta \mid d, \mathcal{H})=\frac{\pi(\Theta \mid \mathcal{H}) \mathcal{L}(d \mid \Theta, \mathcal{H})}{\mathcal{Z}(d \mid \mathcal{H})}
$$

where $\pi(\Theta \mid \mathcal{H})$ is the prior distribution, $\mathcal{L}(d \mid \Theta, \mathcal{H})$ is the likelihood function, and $\mathcal{Z}(d \mid \mathcal{H})$ is the evidence. The posterior $p(\Theta \mid d, \mathcal{H})$ is the target of parameter estimation, and the evidence is the target for hypothesis testing or model selection. As research and development of $3 \mathrm{G}$ instruments ramps up, there is increasing interest in the 
posterior density of gravitational-wave source properties because it is fundamental to answering interesting questions about the astrophysics capabilities of the detectors. However, because of the high cost of computing the posterior density for BNSs, approximate methods are often used to study the capabilities of $3 \mathrm{G}$ detectors; see, e.g., Ref. [11]. Fisher-matrix analyses have been used to approximate the width of $p(\Theta \mid d, \mathcal{H})$, assuming the likelihood is well approximated by a Gaussian distribution. While this assumption is valid for some projections of the posterior, it is not generally valid-even when the SNR is in the thousands-and must be carefully vetted [9]. Hence, a Bayesian treatment of parameter estimates is timely in order to reliably study topics in neutron star astrophysics with networks of $3 \mathrm{G}$ detectors.

In this Letter, we demonstrate how Bayesian inference can be performed on BNS signals using reduced-order models (ROMs) [12-14] of gravitational waveforms. Our work extends previous applications of reduced-order modeling to signals that are up to $90 \mathrm{~min}$ in duration from a low frequency of $5 \mathrm{~Hz}$, which is close to the expected lowfrequency cutoff for $3 \mathrm{G}$ detectors [1,2]. In addition, the ROMs incorporate effects of Earth's rotation on gravitational-wave signals, tidal deformability of neutron stars, and spin-induced orbital precession. We show that our ROMs are accurate representations of the original waveforms at around the $10^{-12}$ level, ensuring that the ROM approximation is valid up to $\mathrm{SNR} \approx 10^{6}$ [15]—good enough for essentially all foreseeable $3 \mathrm{G}$ science.

The ROMs form highly efficient approximations of the likelihood function - the most expensive part of inference. This approximation is known as a reduced-order quadrature (ROQ) $[12,13,16]$. We show that the ROQ can speed up the evaluation of the likelihood function by a factor of around 13000 on individual BNS signals. This makes inference on these signals tractable. Additionally, we show that the ROM and ROQ framework can be applied to accelerate likelihood calculations on multiple overlapping in-band signals. At any given time, there are likely to be hundreds of BNSs in the Universe emitting gravitational waves in the $3 \mathrm{G}$ observing band [17]. However, signals are sufficiently separated in time that they can usually be analyzed separately, though sometimes two or more signals are sufficiently close that a simultaneous analysis is required [4]. We determine the theoretical speedup factor for multiple in-band signals and show that the ROM and ROQ framework can speed up inference by a factor of around 10000 for several overlapping signals.

We perform Bayesian inference on a 90-min-long signal at a similar luminosity distance to GW170817, added into synthetic data of a $3 \mathrm{G}$ network consisting of CE, ET, and a Southern-Hemisphere CE-like detector which we call CE-South. The signal has $\mathrm{SNR} \approx 2400$. We are able to perform Bayesian inference in around 1600 CPU-hours. Without the ROQ, the run-time of the analysis would be around $10^{7}$ CPU-hours. We overcome limitations of the Fisher information matrix by accurately determining the uncertainties of source parameters whose posterior densities are highly non-Gaussian. Our results demonstrate that even loud $3 \mathrm{G}$ signals can be analyzed with modest computational resources. However, reduced-order methods are essential for controlling the computational cost.

The likelihood function and reduced-order models.The most expensive part of evaluating the posterior probability is the likelihood function because it involves computing gravitational waveforms. The log-likelihood function is [18]

$\ln \mathcal{L} \propto-\frac{1}{2}\langle d-h, d-h\rangle=\langle d, h\rangle-\frac{1}{2}\langle h, h\rangle-\frac{1}{2} \mathcal{Z}_{n}$,

where the constant $\mathcal{Z}_{n}=\langle d, d\rangle$ is known as the "noise evidence" and quantifies the likelihood of the data under the hypothesis that they are Gaussian noise [19]. The angle brackets $\langle a, b\rangle$ denote the usual noise-weighted inner product [20].

In the frequency domain, the gravitational waveforms $h$ have the general form [11]

$$
\begin{aligned}
h(f ; \Theta)= & \frac{1}{D_{L}}\left[F_{+}(f ; \xi) h_{+}\left(f ; \theta, \iota, \phi_{c}, t_{c}\right)\right. \\
& \left.+F_{\times}(f ; \xi) h_{\times}\left(f ; \theta, \iota, \phi_{c}, t_{c}\right)\right],
\end{aligned}
$$

where $h_{(+, \times)}\left(f ; \theta, l, \phi_{c}, t_{c}\right)$ are the individual gravitationalwave polarizations and are a function only of the intrinsic parameters $\theta$, orbital inclination $l$, and phase and time at coalescence $\phi_{c}$ and $t_{c}$. $D_{L}$ is the luminosity distance to the source. The quantities $F_{(+, x)}$ are the detector response functions, which depend on the binary's right ascension $\alpha$, declination $\delta$, and polarization phase $\psi$, which define $\xi=(\alpha, \delta, \psi)$. The full set of parameters $\Theta$ which appears on the left-hand side is the combined set of intrinsic and extrinsic parameters, i.e., $\Theta=\left(\theta, \xi, l, \phi_{c}, t_{c}, D_{L}\right)$. The detector response functions are also functions of time and frequency due to Earth's rotation, which cannot be neglected for BNS signals starting from $5 \mathrm{~Hz}$ [11]. Since the time evolution of the response functions is slow compared to that of the gravitational-wave signal, we can use the stationary phase approximation which allows us to separate $F_{(+, x)}$ and $h_{(+, x)}$; see Ref. [11] for details and the explicit form of the frequency-dependent $F_{(+, \times)}$.

In the frequency domain, the ROMs of $h$ [Eq. (3)] have the following general form [13]:

$$
h^{\mathrm{ROM}}(f ; \Theta)=\sum_{J=1}^{N} h\left(\mathcal{F}_{J} ; \Theta\right) B_{J}(f) .
$$

The quantities $B_{J}(f)$ are a basis set which span the space of the signal. The $h\left(\mathcal{F}_{J} ; \Theta\right)$ which appear on the right-hand 
side of Eq. (4) are the unapproximated waveform [Eq. (3)] evaluated at a frequency $\mathcal{F}_{J}$ from a reduced set of $N$ frequencies $\left\{\mathcal{F}_{J}\right\}_{J=1}^{N}$. Previous work on reduced-order modeling for gravitational-wave parameter estimation also constructed ROMs for the waveform amplitude $h^{*} h$ which is used to approximate the $\langle h, h\rangle$ term in the likelihood [Eq. (2)] [13]. We find this unnecessary, and this term can be computed at negligible cost, which we discuss below.

The ROM requires $M / N$ fewer waveform evaluations than the unapproximated expression for the waveform, where $M=T \times\left[f_{\max }-f_{\min }\right]$ and $T$ is the signal duration. This is given by the Nyquist theorem, assuming a fixed sampling rate. For $3 \mathrm{G}$ detectors, we assume $f_{\max }=$ $2048 \mathrm{~Hz}, f_{\min }=5 \mathrm{~Hz}, T=90 \mathrm{~min}(5400 \mathrm{~s})$. When ROMs are substituted into the likelihood function, they form a compressed inner product known as a ROQ. The ROQ speeds up the likelihood function by a factor $[12,13]$

$$
S \approx M / N,
$$

and in general $S \gg 1$. This speedup assumes that the waveforms have a closed-form expression, which frequency-domain waveforms typically do.

ROM construction.-The ROMs are constructed in three steps: (i) make a representative "training space" of gravitational waveforms which span the parameter range of interest, (ii) select basis elements from the training set, and (iii) determine the reduced set of frequency nodes. All steps are achieved using a greedy algorithm [21-23]. We construct ROMs of 90-min-long gravitational-wave signals including spin precession and tidal deformability. That we can build ROMs for 90-min-long BNS signals should not be taken for granted. Previous studies $[12,13,24]$ have not established whether ROMs for such signals can be made in practice or if they would be practical for data analysis. In Refs. [12,13,24], various scalings for ROM basis sizes are given as a function of the low frequency of the signals or parameter-space ranges. However, there has been no systematic study of the size of ROM bases on both low frequency and parameter-space size. The fundamental issue is whether the parameter space can be made small enough to be both effective and efficient for long-duration signals. Below, we show that it is indeed the case.

We focus on individual signals and target a small region of intrinsic parameter space on which we build a training set. The parameters are the chirp mass $\mathcal{M}_{c}$, symmetric mass ratio $\eta$, tidal deformabilities $\left(\lambda_{1}, \lambda_{2}\right)$, spin components projected along the orbital angular momentum axis $\left(\chi_{1}^{z}, \chi_{2}^{z}\right)$, effective-precession spin and the initial value of the azimuthal precession angle $\left(\chi_{p}, \alpha_{0}\right)$, and orbital inclination $l$. We use the waveform model IMRPHENOMPV2-NRTIDALV2 [25-27], which is parametrized by the vector $\theta=\left\{\mathcal{M}, \eta, \chi_{1}^{z}, \chi_{2}^{z}, \chi_{p}, \alpha_{0}, l, \lambda_{1}, \lambda_{2}\right\}$. Inclination appears here because it evolves during the inspiral due to spin-induced orbital precession.
The size of the ROM basis is sensitive to the range in chirp mass. We pick a fiducial chirp-mass value of $\mathcal{M}_{*}=$ $1.385 M_{\odot}$ and restrict the width of the chirp mass of the training set to be $\pm 5 \times 10^{-4} M_{\odot}$. This mass range is approximately $1 \times 10^{3} \Delta \mathcal{M}$, where $\Delta \mathcal{M}$ is the Fishermatrix error estimated using GWBENCH [28]. Following the Fisher-matrix error treatment in Ref. [29], we find that signals with SNRs of around 10 have Fisher errors $\Delta \mathcal{M} \sim 10^{-4} M_{\odot}$. Hence, our chirp-mass range ensures that we can analyze signals with SNRs around 10 without artificially railing against prior bounds in mass. In practice, we may want to use broader priors in mass than are possible with a single parameter-space patch. Broader prior ranges can be employed simply by utilizing multiple ROM bases that individually span small parameter-space ranges. Around 1000 such patches in $\mathcal{M}-\eta$ would be needed to cover the full BNS mass space, assuming BNSs have chirp masses approximately in the range $1 M_{\odot}-2 M_{\odot}$.

All other intrinsic parameters are chosen to have physically motivated ranges. The symmetric mass ratio is restricted to $0.2 \leq \eta \leq 0.25$. Assuming a minimum neutron star mass of $1 M_{\odot}$, this range ensures we describe neutron stars with masses up to $2.6 M_{\odot}$ (around the maximum plausible nonrotating neutron star mass [30-32]). For all other parameters, we consider the following ranges: $0<\chi_{p} \leq 0.1,-0.1 \leq \chi_{1,2}^{z} \leq 0.1,0 \leq \lambda_{1,2} \leq 5000$. Additionally, the ROM is constructed to be valid for all values of sky location parameters $\xi=(\alpha, \delta, \psi)$, luminosity distance $D_{L}$, phase at coalescence $\phi_{c}, l$, and $\alpha$. We consider three starting frequencies $f_{\min }=5,10,20 \mathrm{~Hz}$, maximum frequency $f_{\max }=2048 \mathrm{~Hz}$, and signal duration of $T=90 \mathrm{~min}$. These values of $f_{\min }$ test how the size of the ROM bases scales with the low-frequency cutoff.

We construct a training set of waveforms for the parameter space defined above. The basis and reduced frequency nodes are selected using a greedy algorithm. Details about the training set and greedy algorithm are described in the Supplemental Material [33]. For signals starting in band from $5,10,20 \mathrm{~Hz}$, the ROMs have $N=522,291,179$ basis elements. The basis size only increases by a factor of 3 when going from 20 to $5 \mathrm{~Hz}$, despite the signals being over 40 times longer in duration. Bases of around 500 elements should be typical for ROMs of BNS signals starting from $5 \mathrm{~Hz}$ with parameter ranges similar to those used here. Reducing the chirp mass to that of a $1 M_{\odot}+1 M_{\odot}$ binary will change the signal duration only by a factor of 2 , much less than the difference in the duration of signals starting from 20 vs $5 \mathrm{~Hz}$. Hence, the basis size should be roughly constant for lower-mass systems.

The computational cost of building the ROM is relatively small. We require 160 16-core $2.20 \mathrm{GHz}$ Intel Xeon E5-2660 CPUs running for around $7 \mathrm{~min}$, and then a single CPU running for around $2 \mathrm{~h}$ to complete the basis construction; see step (ii) of the ROM building strategy in the Supplemental Material [33]. The memory footprint of 
the basis is around $90 \mathrm{~GB}$. Thus, it would be feasible to build reduced-order models covering the full chirp-mass range of BNSs.

Likelihood speedup.-The most efficient use of ROMs in Bayesian inference is to compress the large inner products in the likelihood function. The compressed inner products are known as a ROQ integration rule. We obtain the ROQ likelihood by substituting the ROM [Eq. (4)] into the likelihood [Eq. (2)]. The ROQ likelihood is

$$
\ln \mathcal{L}_{\mathrm{ROQ}} \propto L(\Theta)-\frac{1}{2} Q(\Theta)-\frac{1}{2} \mathcal{Z}_{n}
$$

where the functions $L(\Theta)$ and $Q(\Theta)$ are given by

$$
\begin{gathered}
L(\Theta)=\Re \sum_{J=1}^{N} h\left(F_{J} ; \Theta\right) \omega_{J}\left(t_{c}\right), \\
Q(\Theta)=\sum_{I=1}^{N} \sum_{J=1}^{N} h^{*}\left(F_{I} ; \Theta\right) h\left(F_{J} ; \Theta\right) \psi_{I J} .
\end{gathered}
$$

The quantities $\omega_{J}\left(t_{c}\right)$ and $\psi_{I J}$ are integration weights that depend only on the basis functions, data, and noise power spectral density, and are defined in the Supplemental Material [33].

The computational cost of the ROQ likelihood scales as

$$
\ln \mathcal{L} \sim \mathcal{O}(N \times W),
$$

where $W$ is the number of operations required to evaluate the waveform at a given frequency. Unlike previous work [13], we have chosen to write the $Q$ term without the use of an explicit basis for the waveform amplitude $h^{*} h$. The scaling of Eq. (8) is independent of $W$ because waveforms at the reduced frequencies have already been computed as part of $L$ [Eq. (7)]. Thus, Eq. (8) scales like $\sim O\left(N^{2}\right)$, and we find that $N$ is small enough such that $N^{2} \ll N \times W$. For our basis starting from $5 \mathrm{~Hz}$ (which contains $N=522$ basis elements), the theoretical speedup [Eq. (5)] is $S=5400 \mathrm{~s} \times(2048-5) \mathrm{Hz} / 522 \approx 21000$. Empirically, we find a speedup of around 13000 . The degradation in performance is due to fixed overheads, such as allocating data structures for the waveforms. The integration weights $\omega_{I J}\left(t_{c}\right)$ and $\psi_{I J}$ are dependent on the data and noise power spectral density and have to be computed before data analysis can take place. The cost of computing both is negligible in practice.

Validation and accuracy.-The accuracy of the ROQ likelihood [Eq. (6)] is limited by the accuracy of the ROM. We validate the accuracy by computing the mismatch $M$ between the ROM representation of $h$ [Eq. (4)] and its unapproximated form [Eq. (3)]:

$$
M(h)=1-\frac{\left\langle h^{\mathrm{ROM}}, h\right\rangle}{\sqrt{\left\langle h^{\mathrm{ROM}}, h^{\mathrm{ROM}}\right\rangle\langle h, h\rangle}} .
$$

In the noise-weighted inner products, we assume a flat power spectrum, meaning our mismatches are more conservative than if one used a gravitational-wave detector noise power spectral density. In Fig. 1, we show the mismatch $M(h)$ for $2 \times 10^{6}$ random parameter values $\Theta$ that were not included in the training space. We include random sky locations, inclinations, luminosity distances, and phases at coalescence. We also include the frequency-dependent response functions in the mismatch calculations, demonstrating that the ROM is accurate describing signals with amplitudes modulated by Earth's rotation. The mismatch is strongly peaked around $10^{-12}$, ensuring that parameter estimation will be unbiased up to $\mathrm{SNR} \approx 10^{6}$ (so that twice the mismatch multiplied by the SNR squared is less than unity $[15,34])$.

Inference with a high-SNR signal.-As an illustrative example, we consider inference on a signal, which we nickname GW370817. The parameters are $\Theta_{370817}=$ $\left\{\mathcal{M}=1.3854 M_{\odot}, \eta=0.24925, \quad \chi_{1}^{z}=-0.0113, \quad \chi_{2}^{z}=\right.$ $0.01070, \chi_{p}=0.03, \alpha_{0}=1.1, l=0.785, \lambda_{1}=422.5, \lambda_{2}=$ $839.4, D_{L}=38.77 \mathrm{Mpc}, \alpha=1 \mathrm{~h} 57 \min 20.5 \mathrm{~s}, \delta=-14.9$ $\left.\operatorname{deg}, \psi=2.012, \phi_{c}=0\right\}$. This signal has a luminosity distance consistent with GW170817. We add the signal into synthetic data of a three-detector network consisting of CE, ET, and CE-South. We use a "zero-noise" realization of Gaussian noise [35], which (statistically) is the most likely realization. This noise realization has the added convenience that if we use flat priors, the posterior peaks at exactly the true parameter values which serves as a useful diagnostic check. The signal has $\mathrm{SNR}=2400$. We use flat priors on all parameters, and the ranges are given by the range of validity of the ROM. In addition, we impose a physically motivated prior constraint on the component tidal deformability: $\lambda_{2}>\lambda_{1}$. Lastly, we use a uniform prior over a $0.2 \mathrm{~s}$ interval centered on the true trigger time. In

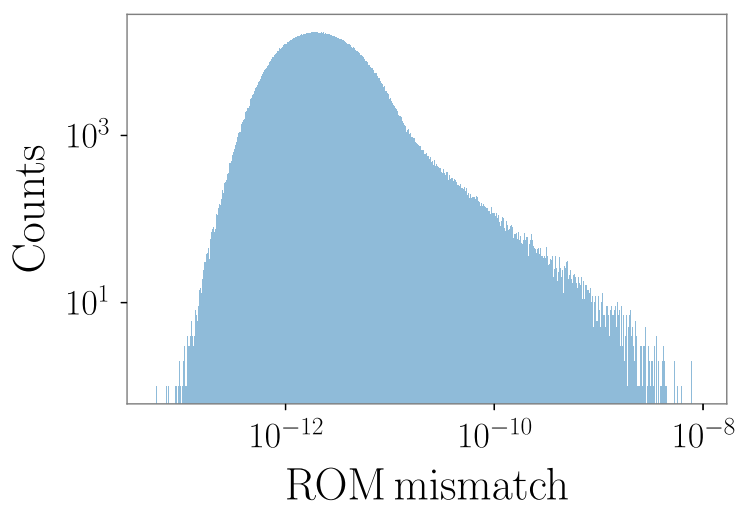

FIG. 1. Accuracy of the ROM approximation for 90-min-long BNS signals starting from $5 \mathrm{~Hz}$. The signals include amplitude modulation due to Earth's rotation. 
general, it is not necessary to restrict the chirp mass prior to such a narrow range. Provided that ROMs exist in local patches covering an extended chirp-mass region, a wide prior can be utilized by building ROQ weights from multiple ROM bases.

We use the DYNESTY nested sampling package to infer the posterior density. In order to obtain well-converged posteriors, we set the number of live points to 5000, and use a random-walk proposal from the BILBY $[10,36]$ inference library, which takes a number of steps equal to 70 times the running estimate of the autocorrelation length. The analysis is parallelized over 160 cores. The analysis takes $10 \mathrm{~h}$ (1600 CPU-hours) on a cluster of 16-core $2.20 \mathrm{GHz}$ Intel Xeon E5-2660 CPUs. The large CPU time occurs because the implementation of the nested sampling algorithm in Refs. $[10,36]$ is extremely slow to converge when the SNR is in the thousands. However, only a handful of events are likely to be detected at these SNRs, with the vast majority of signals having "moderate" SNRs less than 100. Analysis of signals with moderate SNRs takes on the order of a day using a single CPU using ROM or ROQ techniques [13]. In contrast, the CPU time without ROM or ROQ methods would be on the order of $20 \times 10^{6} \mathrm{~h}$, i.e., prohibitively expensive. This analysis highlights the need for improvements to the convergence of stochastic-sampling-based approaches to inference.

In Fig. 2, we show the one- and two-dimensional posterior densities for the component masses and tidal

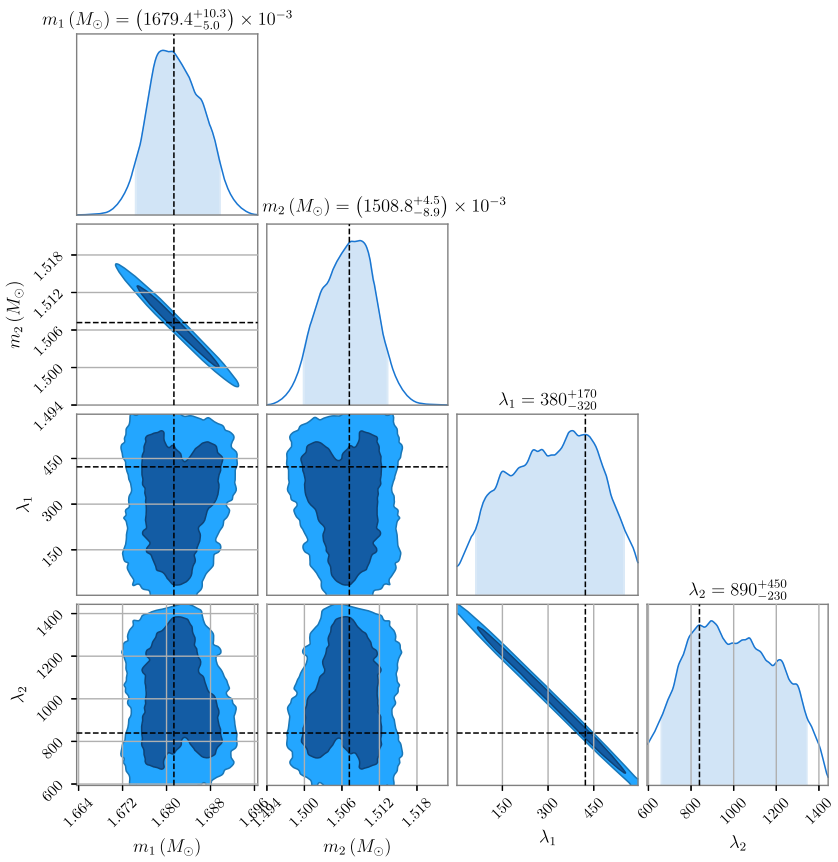

FIG. 2. One- and two-dimensional posterior densities for component masses and component tidal deformabilities. Dark and light shading indicates the one-sigma and two-sigma credible interval, respectively. True parameter values are indicated by dashed lines. deformabilities. The component masses can be constrained to the $\sim 5 \times 10^{-3} M_{\odot}$ level at the $90 \%$ credible intervals, which is consistent with Fisher information estimates. The tidal deformabilities have broad uncertainty and are highly non-Gaussian, demonstrating the importance of full Bayesian inference for understanding how well tidal effects - and hence, nuclear physics — can be constrained by $3 \mathrm{G}$ observatories.

Overlapping signals.-When multiple signals are present simultaneously, the log-likelihood function is

$$
\begin{aligned}
\ln \mathcal{L} & \propto-\frac{1}{2}\left\langle\left(d-\sum_{i=1}^{n} h_{i}\right),\left(d-\sum_{j=1}^{n} h_{j}\right)\right\rangle \\
& =\left\langle d, \sum_{i=1}^{n} h_{i}\right\rangle-\frac{1}{2} \sum_{i j}\left\langle h_{i}, h_{j}\right\rangle-\frac{1}{2} \mathcal{Z}_{n},
\end{aligned}
$$

where the sums run over the independent gravitationalwave signals. The double sum $\sum_{i j}$ runs over all pairs $(i, j)$. In the multiple-signal case, the ROQ likelihood and its scaling are

$$
\begin{aligned}
\ln \mathcal{L}_{\mathrm{ROQ}}= & \sum_{i=1}^{n} L\left(\Theta_{i}\right)-\sum_{i=1}^{n} \frac{1}{2} Q\left(\theta_{i}\right) \\
& +\sum_{\{j, k\}}^{n^{2} / 2} R_{j k}\left(\Theta_{j}, \Theta_{k}, \Delta t^{j k}\right)-\frac{1}{2} \mathcal{Z}_{n} \\
\sim & \mathcal{O}\left(N \times n \times W+N^{2} \times\left(n+n^{2} / 2\right)\right),
\end{aligned}
$$

where $L$ and $Q$ are given by Eqs. (7) and (8). The $R_{j k}$ term sums over products of all pairs of waveforms $\{j, k\}$, with $j>k$, and is also a function of the relative time offset between two signals, $\Delta t^{j k}$. The function $R_{j k}\left(\Theta_{j}, \Theta_{k}, \Delta t^{j k}\right)$ is

$$
\begin{aligned}
\left\langle h\left(\Theta_{j}\right), h\left(\Theta_{k}\right)\right\rangle & \approx R_{j k}\left(\Theta_{j}, \Theta_{k}, \Delta t^{j k}\right) \\
& =\Re \sum_{K=1}^{N} \sum_{L=1}^{N} h^{*}\left(F_{K} ; \Theta_{j}\right) h\left(F_{L} ; \Theta_{k}\right) \Gamma_{K L}\left(\Delta t^{j k}\right),
\end{aligned}
$$

where the matrix $\Gamma_{K L}\left(\Delta t^{j k}\right)$ is a set of integration weights given in the Supplemental Material [33]. The overall speedup of the multiple-signal ROQ likelihood Eq. (12) with respect to non-ROQ likelihood [Eq. (11)] is

$$
S \approx \frac{M n W+M n^{2} / 2}{N n W+N n^{2} / 2}, \quad \text { with } \quad n>1,
$$

where we have kept terms at $\mathcal{O}(W)$ and $\mathcal{O}\left(n^{2}\right)$. The $N^{2} n^{2}$ scaling is potentially problematic if the number of in-band signals is large. However, most overlapping signalsroughly between $96 \%$ and $99.5 \%$ - are well separated in time so that they can be analyzed separately (Tables I and IV 
of Ref. [4]). Thus, we only consider the speedup for the simultaneous analysis of a few in-band signals. We empirically determine a speedup of $\sim \mathcal{O}\left(10^{4}\right)$ compared to the calculation time without ROM for up to ten in-band signals. We show the speedup as a function of the number of in-band signals in the Supplemental Material [33].

Discussion.-Reduced-order models of gravitationalwave signals from BNS mergers can be used to accelerate parameter estimation in $3 \mathrm{G}$ observatories, thereby removing a computational hurdle. This work lays the groundwork for detailed studies of BNS systems in the $3 \mathrm{G}$ era. Further, the ROM and ROQ framework can be used to efficiently carry out inference on data containing multiple overlapping signals. Further avenues to pursue include ROMs of more sophisticated waveforms, e.g., with higher-order gravitational-wave modes, which can place tighter constraints on parameter estimates [37]. Bayesian inference on very loud signals-SNR $\sim \mathcal{O}(1000)$-requires significantly more likelihood evaluations than in analyses of LIGO and Virgo signals. Sampling-based methods for Bayesian inference will have to be significantly adapted and scaled up in order to efficiently analyze data in the $3 \mathrm{G}$ era. For instance, Hamiltonian Monte Carlo methods [38] (which exploit gradients of posterior densities) and machine learning techniques, e.g., Refs. [39-42] (which provide rapid approximations to reduced-order models and posterior densities) may be promising avenues to explore.

This work was supported through Australian Research Council Centre of Excellence Grant No. CE170100004. P. L. is a recipient of the Australian Research Council Future Fellowship No. FT160100112 and Discovery Project No. DP180103155. I. M. is a recipient of the Australian Research Council Future Fellowship No. FT190100574. E. T. is a recipient of the Australian Research Council Future Fellowship No. FT150100281. S. V. acknowledges support of the National Science Foundation through the NSF Grant No. PHY-1836814. We greatly appreciate suggestions and comments from members of the LIGO and Virgo Collaborations and Cosmic Explorer Consortium. In particular, Leo Tsukada, Nathan Johnson-McDaniel, Philippe Landry, and Aidan Brooks. This manuscript has LIGO Document No. P2100051. S. B. and B. S. acknowledge the support from NSF grant PHY-1836779. B.S. is supported by NSF grants No. AST-2006384 and No. PHY-2012083. S. E. F. is partially supported by NSF grants PHY-1806665 and DMS-1912716.

*rory.smith@monash.edu

†sub284@psu.edu

"bss25@psu.edu

[1] D. Reitze, R. X. Adhikari, S. Ballmer, B. Barish, L. Barsotti, G. Billingsley, D. A. Brown, Y. Chen, D. Coyne, R. Eisenstein et al., Bull. Am. Astron. Soc. 51, 035 (2019).
[2] M. Maggiore, C. V. D. Broeck, N. Bartolo, E. Belgacem, D. Bertacca, M. A. Bizouard, M. Branchesi, S. Clesse, S. Foffa, J. Garca-Bellido et al., J. Cosmol. Astropart. Phys. 03 (2020) 050.

[3] V. Baibhav, E. Berti, D. Gerosa, M. Mapelli, N. Giacobbo, Y. Bouffanais, and U. N. Di Carlo, Phys. Rev. D 100, 064060 (2019).

[4] A. Samajdar, J. Janquart, C. van den Broeck, and T. Dietrich (to be published).

[5] S. Vitale and M. Evans, Phys. Rev. D 95, 064052 (2017).

[6] R. X. Adhikari, P. Ajith, Y. Chen, J. A. Clark, V. Dergachev, N. V. Fotopoulos, S. E. Gossan, I. Mandel, M. Okounkova, V. Raymond et al., Classical Quantum Gravity 36, 245010 (2019).

[7] J. Veitch, V. Raymond, B. Farr, W. Farr, P. Graff, S. Vitale, B. Aylott, K. Blackburn, N. Christensen, M. Coughlin et al., Phys. Rev. D 91, 042003 (2015).

[8] G. Ashton et al., Astrophys. J. Suppl. Ser. 241, 27 (2019).

[9] M. Vallisneri, Phys. Rev. D 77, 042001 (2008).

[10] R. J. E. Smith, G. Ashton, A. Vajpeyi, and C. Talbot, Mon. Not. R. Astron. Soc. 498, 4492 (2020).

[11] W. Zhao and L. Wen, Phys. Rev. D 97, 064031 (2018).

[12] P. Canizares, S. E. Field, J. Gair, V. Raymond, R. Smith, and M. Tiglio, Phys. Rev. Lett. 114, 071104 (2015).

[13] R. Smith, S. E. Field, K. Blackburn, C.-J. Haster, M. Pürrer, V. Raymond, and P. Schmidt, Phys. Rev. D 94, 044031 (2016).

[14] M. Prrer, Classical Quantum Gravity 31, 195010 (2014).

[15] L. Lindblom, B. J. Owen, and D. A. Brown, Phys. Rev. D 78, 124020 (2008).

[16] H. Antil, S. E. Field, F. Herrmann, R. H. Nochetto, and M. Tiglio, J. Sci. Comput. 57, 604 (2013).

[17] B. P. Abbott et al. (LIGO Scientific and Virgo Collaborations), Phys. Rev. Lett. 120, 091101 (2018).

[18] B. J. Owen and B. S. Sathyaprakash, Phys. Rev. D 60, 022002 (1999).

[19] B. P. Abbott, R. Abbott, T. D. Abbott, S. Abraham, F. Acernese, K. Ackley, C. Adams, V. B. Adya, C. Affeldt et al., Living Rev. Relativity 23, 3 (2020).

[20] J. Veitch and A. Vecchio, Phys. Rev. D 81, 062003 (2010).

[21] H. Antil, D. Chen, and S. Field, Comput. Sci. Eng. 20, 10 (2018).

[22] S. E. Field, C. R. Galley, J. S. Hesthaven, J. Kaye, and M. Tiglio, Phys. Rev. X 4, 031006 (2014).

[23] M. Barrault, Y. Maday, N. C. Nguyen, and A. T. Patera, C. R. Math. 339, 667 (2004).

[24] S. Morisaki and V. Raymond, Phys. Rev. D 102, 104020 (2020).

[25] T. Dietrich, S. Khan, R. Dudi, S. J. Kapadia, P. Kumar, A. Nagar, F. Ohme, F. Pannarale, A. Samajdar, S. Bernuzzi et al., Phys. Rev. D 99, 024029 (2019).

[26] T. Dietrich, S. Bernuzzi, and W. Tichy, Phys. Rev. D 96, 121501(R) (2017).

[27] T. Dietrich, A. Samajdar, S. Khan, N. K. JohnsonMcDaniel, R. Dudi, and W. Tichy, Phys. Rev. D 100, 044003 (2019).

[28] S. Borhanian, arXiv:2010.15202.

[29] E. Poisson and C. M. Will, Phys. Rev. D 52, 848 (1995). 
[30] R. Abbott, T. D. Abbott, S. Abraham, F. Acernese, K. Ackley, C. Adams, R. X. Adhikari, V. B. Adya, C. Affeldt, M. Agathos et al., Astrophys. J. 896, L44 (2020).

[31] R. Essick, P. Landry, and D. E. Holz, Phys. Rev. D 101, 063007 (2020).

[32] Y. Lim and J. W. Holt, Eur. Phys. J. A 55, 209 (2019).

[33] See Supplemental Material at http://link.aps.org/ supplemental/10.1103/PhysRevLett.127.081102 for a description of the greedy algorithm for finding the reduced basis elements and interpolation nodes, as well as the speedup as a function of the number of in-band signals.

[34] F. Ohme, Classical Quantum Gravity 29, 124002 (2012).

[35] C. Pankow, K. Chatziioannou, E. A. Chase, T. B. Littenberg, M. Evans, J. McIver, N. J. Cornish, C.-J. Haster, J. Kanner, V. Raymond et al., Phys. Rev. D 98, 084016 (2018).
[36] G. Ashton, M. Hbner, P. D. Lasky, C. Talbot, K. Ackley, S. Biscoveanu, Q. Chu, A. Divakarla, P. J. Easter, B. Goncharov et al., Astrophys. J. Suppl. Ser. 241, 27 (2019).

[37] J. C. Bustillo, T. Dietrich, and P. D. Lasky, Astrophys. J. Lett. 912, L10 (2021).

[38] M. Betancourt, arXiv:1701.02434.

[39] H. Gabbard, C. Messenger, I. S. Heng, F. Tonolini, and R. Murray-Smith, arXiv:1909.06296.

[40] H. Shen, E. A. Huerta, Z. Zhao, E. Jennings, and H. Sharma, arXiv:1903.01998.

[41] A. J. K. Chua and M. Vallisneri, Phys. Rev. Lett. 124, 041102 (2020).

[42] A. J. K. Chua, C. R. Galley, and M. Vallisneri, Phys. Rev. Lett. 122, 211101 (2019). 